\title{
Production and characterization of the bread wheat lines with introgression of chromosome 6Agi2 Thynopirum intermedium
}

Ivanova Yu.N.*, Stasyuk A.I., Skolotneva E.S., Silkova O.G.

Institute of Cytology and Genetics, SB RAS, Novosibirsk, Russia

*email: kabanenko@bionet.nsc.ru

Wheatgrass Thinopyrum intermedium is a source of agronomically valuable traits for common wheat. In breeding programs, partial wheat-wheatgrass amphidiploids and lines with wheatgrass chromosome substitution are widely used. The line Agis 1 (6Agi2/6D) is included in the cultivar Tulaykovskaya 10 (T10) pedigree. The wheatgrass chromosome 6Agi2 carries complex resistance to fungal diseases in various ecologicalgeographical zones and does not reduce the yield and grain quality. Varieties with 6Agi2/6D substitution are used in breeding programs. In this work, in hybrid combinations of cultivar T10 with cultivars Novosibirskaya 31 (H31) and Saratovskaya 29 (C29), plants with 6Agi2/6D chromosome substitution were isolated and their offspring were assessed for economically valuable traits. To identify chromosome 6Agi2, we used DNA markers specific to the long and short arms of this chromosome, as well as genomic in situ hybridization. Analysis of meiosis showed the cytological stability of the new forms; bivalents were formed in all meiocytes by both wheat chromosomes and wheatgrass chromosomes. According to the results of the field experiment in 2020, the samples N31xT10 and T10xS29 was characterized by high productivity traits. The mass of grains per plant and the number of grains per plant did not significantly differ from the parent varieties and the N31 variety. T10xS29 plants were characterized by the ability to tie $3.77 \pm 0.1$ grains per spike, the range of variability of the trait was from 2.93 to 4.62 in individuals. The protein content in the grain was $17.91 \%$, the gluten content was $40.55 \%$. According to the screening for fungal diseases resistance carried out in the field of 2018 and 2020, chromosome 6Agi2 preserves immunity in plants to the West Siberian population of brown rust and to dominant races of stem rust, also provides medium resistant and medium susceptible types of reaction to yellow rust.

Acknowledgements: This work was supported by the grant of the Russian Science Foundation No. 21-76-30003 and the budget project No. 0259-2021-0012. 